\title{
Dimerization of signalling modules of the EvgAS and BvgAS phosphorelay systems
}

\author{
Anne-Laure Perraud a, Karsten Rippe ${ }^{\mathrm{b}}$, Marcus Bantscheff c, Michael Glocker ${ }^{\mathrm{c}}$, \\ Magnus Lucassen d, Kirsten Jung d, Walter Sebald e, Verena Weiss ${ }^{\mathrm{f}}$, Roy Gross a,* \\ a Lehrstuhl für Mikrobiologie, Biozentrum, Universität Würzburg, Am Hubland, D-97074 Würzburg, Germany \\ b Deutsches Krebsforschungszentrum, Abteilung Biophysik der Makromoleküle, D-69115 Heidelberg, Germany \\ c Fakultät für Chemie, Universität Konstanz, D-78457 Constanz, Germany \\ d Abteilung Mikrobiologie, Universität Osnabrück, D-46069 Osnabrück, Germany \\ e Lehrstuhl für Physiologische Chemie II, Biozentrum, Universität Würzburg, D-97074 Würzburg, Germany \\ f Fakultät für Biologie, Universität Konstanz, D-78434 Constanz, Germany
}

Received 13 December 1999; received in revised form 28 February 2000; accepted 9 March 2000

\begin{abstract}
Biophysical and biochemical properties of signalling proteins or domains derived from the unorthodox EvgAS and BvgAS two-component phosphorelay systems of Escherichia coli and Bordetella pertussis were investigated. Oligomerization of the effector proteins EvgA and BvgA and of truncated EvgS and BvgS derived signalling proteins containing the receiver and histidine containing phosphotransfer (HPt) domains or comprising only the HPt domains were characterized by native gel electrophoresis, gel permeation experiments and analytical ultracentrifugation. The results obtained by the different methods are consistent with non-phosphorylated $\mathrm{EvgA}$ and BvgA proteins being dimers in solution with a dissociation constant significantly below $1 \mu \mathrm{M}$. In contrast, all sensor derived domains of EvgS and BvgS were observed to be monomers in vitro. No indications for a phosphorylation induced stimulation of oligomerization of the C-terminal histidine kinase domains could be detected. In agreement with these data, surface plasmon resonance studies revealed a 2:1 stoichiometry in the interaction of EvgA with the immobilized EvgS HPt domain and an affinity constant of $1.24 \times 10^{6} \mathrm{M}^{-1}$. C 2000 Elsevier Science B.V. All rights reserved.
\end{abstract}

Keywords: Analytical ultracentrifugation; Surface plasmon resonance; Dimerization; Phosphorelay; BvgAS; EvgAS; Mass spectrometry

\section{Introduction}

Two-component systems are found in eubacteria, archaea, several lower eukaryotic organisms and plants [1-3]. Signals are perceived by a sensory his-

\footnotetext{
* Corresponding author. Fax: +49-931-888-4402; http://www.biozentrum.uni-wuerzburg.de/microbiology/; E-mail: roy@biozentrum.uni-wuerzburg.de
}

tidine kinase, which, after autophosphorylation at a histidine residue in the conserved transmitter domain, serves as a phosphodonor for phosphorylation of further signalling domain(s). In classical two-component systems, a one-step phosphotransfer occurs from the phosphohistidine in the transmitter to an aspartic acid residue in the receiver domain of the effector protein. Phosphorylation of the effector protein has regulatory consequences for its biological activity. For instance, many two-component effector 
proteins are transcription factors, which become activated by phosphorylation [4]. Instead of the phosphohistidine presented by the histidine kinases, compounds such as acetyl phosphate may be used as phosphodonors for the receiver domains of the effector proteins in vitro and in some cases possibly also in vivo $[5,6]$. This demonstrates that the receiver domains of the effector proteins harbor the enzymatic activity for their phosphorylation. On the basis of sequence similarities and differences in their domain structures, the effector proteins can be classified into several subfamilies [4].

In 'unorthodox' two-component systems, a multistep phosphorelay occurs between alternating histidine and aspartate residues (His-Asp-His-Asp) before the effector proteins are activated [2]. These systems contain additional signalling modules intercalated between the transmitter domain of the sensor protein and the receiver domain of the effector protein. These additional domains consist of a second 'intermediate' receiver domain and the so-called histidine containing phosphotransfer ( $\mathrm{HPt})$ domain [7]. The intermediate receiver domains appear to be highly related to the receiver domains found in the effector proteins. The various phosphorelay domains are either present as independent proteins or combined in various combinations in complex multidomain proteins [3]. For example, in the case of the Bvg phosphorelay system of Bordetella pertussis, the etiological agent of whooping cough, and of the highly related Evg phosphorelay system of Escherichia coli three domains, the transmitter, the intermediate receiver and the HPt domain, are fused in the single multidomain sensor proteins $\mathrm{BvgS}$ and EvgS, respectively [8-10]. The Bvg system of B. pertussis is known to be the master regulator of virulence controlling the expression of several toxins and adhesins [11]. Nothing is known so far about the function of the Evg system in E. coli. However, in agreement with the extensive sequence conservation between the two systems, both appear to perceive the same environmental signals which include sulfate ions and temperature $[8,9]$.

The structural consequences of phosphorylation for the signal transduction proteins are not well understood. Oligomerization of signalling domains appears to be an important feature during signal transduction by two-component systems. For exam- ple, for several effector proteins it has been reported that phosphorylation induces dimerization and/or cooperative binding to the respective target sites on the DNA [12-16]. Similarly, histidine kinases are able to dimerize and autophosphorylation of the histidine kinases in fact may occur between two homodimeric protein partners [17]. Genetic and biochemical evidence indicated that the $\mathrm{BvgA}$ and $\mathrm{BvgS}$ proteins of $B$. pertussis, the etiological agent of whooping cough, also have dimerizing properties [9,18-20].

In the present report we attempted to gain more insight in the molecular basis of signal transduction in the Bvg and Evg phosphorelay systems of B. pertussis and E. coli. We investigated the dimerization and autophosphorylation properties of several signalling domains derived from the unorthodox histidine kinases EvgS and BvgS and of their cognate effector proteins EvgA and BvgA.

\section{Materials and methods}

\subsection{Bacterial strains, plasmids and growth conditions}

The bacterial strains and plasmids used in this study are listed in Table 1. E. coli DH5 $\alpha$ was used as a host for gene cloning and E. coli M15 and SF120 for overexpression of proteins as described previously [21,22]. The bacteria were grown either in Luria-Bertani (LB) liquid broth or on LB-agar plates. When necessary antibiotics were added to the following final concentrations: ampicillin, 100 $\mu \mathrm{g} / \mathrm{ml}$; kanamycin, $50 \mu \mathrm{g} / \mathrm{ml}$.

\subsection{Overexpression and purification of two-component proteins}

The proteins analyzed are shown in Fig. 1. For purification the genes encoding the response regulators EvgA and BvgA were cloned in the expression vector pCYTEXP1 [22] resulting in plasmids pCYTEvgA and pCYT-BvgA [23]. The native proteins were then overproduced as described [23]. The open reading frames encoding the sensor derived domains EvgS-RO, EvgS-O, BvgS-RO and BvgS-O were cloned in the pQE30 and pQE31 vectors (Qiagen) creating an N-terminal His-tag and resulting in plasmids pQE-EvgS-RO, pQE-EvgS-O, pQE-BvgS-RO 
Table 1

Strains and plasmids

\begin{tabular}{|c|c|c|}
\hline Bacterial strains and plasmids & Relevant features & Source \\
\hline E. coli $\mathrm{DH} 5 \alpha$ & strain for high efficiency transformation & Gibco \\
\hline E. coli $\mathrm{M} 15$ & strain for cloning and overproducing His-tagged proteins & Qiagen \\
\hline E. coli $\mathrm{SF} 120$ & strain for overexpression of proteins & [21] \\
\hline pQE30 and pQE31 & expression vectors for $\mathrm{N}$-terminal His-tag cloning & Qiagen \\
\hline pCYTEXP1 & expression vector & {$[22]$} \\
\hline pQE-BvgS-RO & $\begin{array}{l}\text { derivative of pQE30 expressing the His-tagged BvgS receiver and output }(\mathrm{HPt}) \\
\text { domains }\end{array}$ & [23] \\
\hline pQE-BvgS-O & derivative of pQE30 expressing the His-tagged BvgS output $(\mathrm{HPt})$ domain & {$[23]$} \\
\hline pQE-EvgS-RO & derivative of $\mathrm{pQE} 30$ expressing the His-tagged EvgS receiver and HPt domains & {$[23]$} \\
\hline pQE-EvgS-O & derivative of pQE30 expressing the His-tagged EvgS HPt domain & {$[23]$} \\
\hline pCYT-BvgA & derivative of pCYTEXP1 expressing BvgA & {$[23]$} \\
\hline pCYT-EvgA & derivative of pCYTEXP1 expressing EvgA & {$[23]$} \\
\hline
\end{tabular}

and pQE-BvgS-O, respectively (Table 1). The proteins were overexpressed in E. coli M15 and purified by affinity chromatography according to the manufacturer's protocol (Qiagen). The detailed purification protocols of the various proteins were described recently [23].

\subsection{Biotinylation of EvgS-O and EvgS-RO}

The two proteins EvgS-O and EvgS-RO were biotinylated using $N$-[biotinyl-6-amino]-capronate- $N$ succinimidylester (Fluka, Buchs, Switzerland) according to manufacturers instructions. A stock solution of biotinylation reagent $(0.5 \mu \mathrm{g} / \mu \mathrm{l})$ in dimethylformamide was freshly prepared prior to reaction. Proteins $(0.3 \mu \mathrm{g} / \mu \mathrm{l})$ were dissolved in $50 \mathrm{mM}$ $\mathrm{NaHCO}_{3}$ (pH 8.5). The modification reactions were performed at different protein/reagent ratios $(1: 2$ and $1: 5$, respectively) for $30 \mathrm{~min}$ at $37^{\circ} \mathrm{C}$ and stopped by the addition of glacial acetic acid. Biotinylation sites in the EvgS-RO and EvgS-O proteins were identified by mass spectrometric peptide mapping as described below.

\subsection{Tryptic on-target digestion of biotinylated EvgS-O}

A protein solution $(2 \mu \mathrm{l})$ containing biotinylated EvgS-O was spotted on the MALDI-MS target. Proteolytic on-target digestion was performed after evaporation of the solvent. A freshly prepared trypsin solution $(2 \mu \mathrm{l} ; 0.01 \mu \mathrm{g} / \mu \mathrm{l}$; Sigma, Deisenhofen,
Germany), dissolved in $50 \mathrm{mM} \mathrm{NH} \mathrm{NHCO}_{3}, \mathrm{pH} 8$, was added, resulting in an enzyme/substrate ratio of $1: 100$. The target was placed in a humid atmosphere and was incubated at $37^{\circ} \mathrm{C}$ for $3 \mathrm{~h}$. Subsequently, the solvent was evaporated within $30 \mathrm{~min}$ at room temperature. The on-target digestion products were analyzed by MALDI-MS peptide mapping as described below.

\subsection{Avidin Sepharose affinity isolation of biotinylated EvgS-O peptide}

The biotinylated amino acid residue of EvgS-O was identified using the avidin-biotin chemistry combined with MALDI-MS as described previously $[24,25]$. In brief, the biotinylated EvgS-O was enzymatically digested in $\mathrm{NH}_{4} \mathrm{HCO}_{3}$ buffer, $\mathrm{pH} 7.8$ for $3 \mathrm{~h}$ at $37^{\circ} \mathrm{C}$ using endoprotease Asp-N (Boehringer Mannheim, Mannheim, Germany). The enzyme/substrate ratio was 1:200. Avidin (Sigma, Deisenhofen, Germany) was coupled to NHS-activated Sepharose using standard protocols as suggested by the supplier (Amersham Pharmacia Biotech, Freiburg, Germany). Three $\mu 1$ of avidin coupled Sepharose suspension in PBS buffer ( $30 \mathrm{mM}$ sodium phosphate, $3 \mathrm{M} \mathrm{NaCl}$, $\mathrm{pH}$ 7.2) was added to $5 \mu$ l of the digestion mixture. The suspension was vortexed for approximately $10 \mathrm{~min}$, centrifuged to settle the Sepharose and the supernatant was discarded. Several washing steps were carried out with PBS $(1 \times 50 \mu \mathrm{l})$ followed by deionized water $(2 \times 100 \mu \mathrm{l})$ and finally the pellet was resuspended in $3 \mu \mathrm{l}$ of a solvent mixture consist- 
ing of $0.1 \%$ aqueous trifluoroacetic acid (TFA) and $40 \% \mathrm{CH}_{3} \mathrm{CN}(\mathrm{v} / \mathrm{v})$. This suspension was subjected to mass spectrometric analysis.

\subsection{Mass spectrometry}

MALDI-MS analyses were carried out with a Bruker Biflex linear time-of-flight spectrometer (Bruker-Franzen, Bremen, Germany). For molecular weight determination of the Bvg/Evg proteins $1 \mu \mathrm{l}$ of sample solution was mixed with $0.8 \mu \mathrm{l}$ of a saturated solution of $\alpha$-cyano-4-hydroxy cinnamic acid (HCCA) dissolved in $\mathrm{CH}_{3} \mathrm{CN} / 0.1 \%$ TFA $(2: 1, \mathrm{v} / \mathrm{v})$ directly on the target. After evaporation of the solvent, the matrix/analyte mixture was recrystallized from $1.5 \mu \mathrm{CH}_{3} \mathrm{CN} / 0.1 \%$ TFA $(2: 1, \mathrm{v} / \mathrm{v})$ prior to data acquisition.

In the case of avidin Sepharose affinity separated samples a thin layer of matrix (saturated HCCA solution in acetone) was first placed on the target. After evaporation of the solvent, $1 \mu 1$ of Sepharose slurry was added and allowed to dry. Finally, $0.4 \mu \mathrm{l}$ of a saturated solution of HCCA dissolved in $\mathrm{CH}_{3} \mathrm{CN} / 0.1 \%$ TFA $(2: 1, \mathrm{v} / \mathrm{v})$ was added. The dried Sepharose was blown off by applying a stream of nitrogen gas.

Nano-ESI-MS spectra were recorded on a Vestec A 201 single quadrupole mass spectrometer (PerSeptive Biosystems, Framingham, MA, USA), as reported elsewhere [26,27]. Volumes of $0.5-2 \mu 1$ were loaded by dipping the capillaries into the corresponding sample solutions.

\subsection{Native polyacrylamide gradient gels and gel permeation experiments}

Native polyacrylamide gradient gels were prepared as described previously [28]. The polyacrylamide gradient ranged from 4 to $28 \%$ and was combined with a sucrose gradient ranging from 0 to $20 \%$. Electrophoresis was carried out for $12 \mathrm{~h}$ at $100-150 \mathrm{~V}$. As molecular weight markers the purified proteins of the MW-GF-70 Kit (Sigma) were used which include aldolase $(158 \mathrm{kDa}), \beta$-lactoglobulin $(36.6 \mathrm{kDa})$, anhydrase $(29 \mathrm{kDa})$ and cytochrome $c(12.6 \mathrm{kDa})$. The gel permeation experiments were carried out according to the manufacturer's protocols using a Superdex $75 \mathrm{HR}-10$ FPLC column (Pharmacia) in $\mathrm{NH}_{4} \mathrm{HCO}_{3}$ buffer at $\mathrm{pH}$ 8.5. For molecular weight calibration of the column the proteins of the MW-GH-70 Kit (Sigma) were used once more.

\subsection{Chemical synthesis of acetyl $\left[{ }^{32} P\right]$ phosphate and phosphorylation experiments}

For the synthesis of acetyl $\left[{ }^{32} \mathrm{P}\right]$ phosphate the protocol of Stadtman [29] was modified as follows [30]: $0.19 \mathrm{ml}$ of pyridine, $0.3 \mathrm{ml}$ of $0.33 \mathrm{M} \mathrm{K}_{2} \mathrm{HPO}_{4}$ and $0.1 \mathrm{ml}$ of carrier-free $\left[{ }^{32} \mathrm{P}\right]$ orthophosphate (specific activity $10 \mathrm{mCi} \mathrm{ml}^{-1}$; Amersham Buchler) were stirred on ice in a $16 \times 100$ Pyrex tube. Then $22 \mu 1$ acetic anhydride was added slowly and incubated on ice. $\mathrm{LiOH}(4 \mathrm{~N})$ was added to adjust to $\mathrm{pH} 7$. Finally, acetyl $\left[{ }^{32} \mathrm{P}\right]$ phosphate was precipitated with $4.5 \mathrm{ml}$ of $100 \%$ ethanol for $1 \mathrm{~h}$ on ice. The precipitated acetyl $\left[{ }^{32} \mathrm{P}\right]$ phosphate was collected by centrifugation, washed twice with $5 \mathrm{ml}$ cold ethanol and dried over $\mathrm{KOH}$ and $\mathrm{CaCl}_{2}$. Acetyl $\left[{ }^{32} \mathrm{P}\right]$ phosphate was solved in $180 \mu \mathrm{l}$ of $50 \mathrm{mM}$ Tris- $\mathrm{HCl}, \mathrm{pH} 7.5,5 \%$ glycerol, $0.1 \mathrm{mM}$ EDTA and $1 \mathrm{mM}$ dithiothreitol and used immediately for the phosphorylation assays.

For in vitro phosphorylation with acetyl $\left.{ }^{32} \mathrm{P}\right]$ phosphate the proteins were incubated in $50 \mathrm{mM}$ Tris- $\mathrm{HCl}, \mathrm{pH} 7.5,20 \mathrm{mM} \mathrm{MgCl}_{2}$ and $300 \mathrm{mM} \mathrm{KCl}$ with $20 \mathrm{mM}$ acetyl $\left[{ }^{32} \mathrm{P}\right]$ phosphate at $30^{\circ} \mathrm{C}$. The reaction was stopped using SDS-PAGE sample buffer [31] and subjected to SDS-PAGE. In vitro phosphorylation of proteins with $\left[{ }^{33} \mathrm{P}\right] \gamma \mathrm{ATP}$ was performed as described recently [23]. The phosphorylated proteins were detected using a phosphoimager (Molecular Dynamics).

\subsection{Analytical sedimentation equilibrium ultracentrifugation ( $A U C$ )}

Sedimentation equilibrium experiments were carried out on a Beckman Instruments Optima XL-A analytical ultracentrifuge with absorbance optics. Protein solutions of $A_{280}=0.1-0.2$ (corresponding to concentrations of $10-20 \mu \mathrm{M}$ in terms of protein monomer) in a buffer containing $50 \mathrm{mM}$ Tris- $\mathrm{HCl}$, $\mathrm{pH} 7.5,50 \mathrm{mM} \mathrm{KCl}$ and $4 \%$ glycerol (HK buffer) were analyzed at 10000 and $25000 \mathrm{rpm}$ at $4^{\circ} \mathrm{C}$ with an An60Ti rotor. For measurements of the response regulator proteins BvgA and EvgA the buffer was supplemented with $5 \mathrm{mM} \mathrm{MgCl}_{2}$ (RR buffer). The 
effect of chemical phosphorylation of truncated forms of BvgS and EvgS was studied by the addition of $10 \mathrm{mM}$ acetyl phosphate and $10 \mathrm{mM} \mathrm{MgCl}$. Calculation of the partial specific volume from the amino acid composition of the proteins and analysis of the data by one exponential fit was conducted as described recently [32]. The density $\rho$ of both the HK and RR buffer was measured to be $1.017 \mathrm{~g}$ $\mathrm{ml}^{-1}$ at $4^{\circ} \mathrm{C}$ with a DMA 5000 density meter (Anton Paar, Graz, Austria).

\subsection{Biosensor experiment}

All surface plasmon resonance (SPR) experiments were carried out in a BIAcore 2000 system (Pharmacia Biosensor) at $25^{\circ} \mathrm{C}$ with a flow rate of $10 \mu \mathrm{l}$ $\mathrm{min}^{-1}$ in HBS buffer consisting of $10 \mathrm{mM}$ HEPES, $\mathrm{pH} 7.4,150 \mathrm{mM} \mathrm{NaCl}, 3.4 \mathrm{mM}$ EDTA, $0.005 \%$ surfactant P20 with a data collection rate of $2.5 \mathrm{~s}^{-1}$. To analyze the interaction of EvgA with the HPt domain of EvgS, the biotinylated derivatives of the EvgS-RO and EvgS-O proteins containing either the EvgS receiver and the HPt domain or only the HPt domain, respectively, were coupled to BIAcore streptavidin sensor chips (Pharmacia) at a density of about 1000 resonance units (RU) as described recently [33]. The sensorgrams were generated and evaluated using the BIAevaluation 2.1 software.

\section{Results}

\subsection{Dimerization properties of effector proteins and isolated signalling domains}

The effector proteins EvgA and BvgA and the pre-

Table 2

MALDI-MS molecular weight determinations of effector proteins and signalling modules

\begin{tabular}{lll}
\hline Protein & $M_{\mathrm{r}}$ & MW (experimental) $^{\mathrm{a}}$ \\
\hline BvgA & 22952 & 22949 \\
BvgS-RO & 30892 & 30915 \\
BvgS-O & 17279 & 17275 \\
EvgA & 22690 & 22693 \\
EvgS-RO & 31115 & 31120 \\
EvgS-O & 16127 & 16126 \\
\hline
\end{tabular}

${ }^{\mathrm{a}}$ Confirmed by ESI-MS.

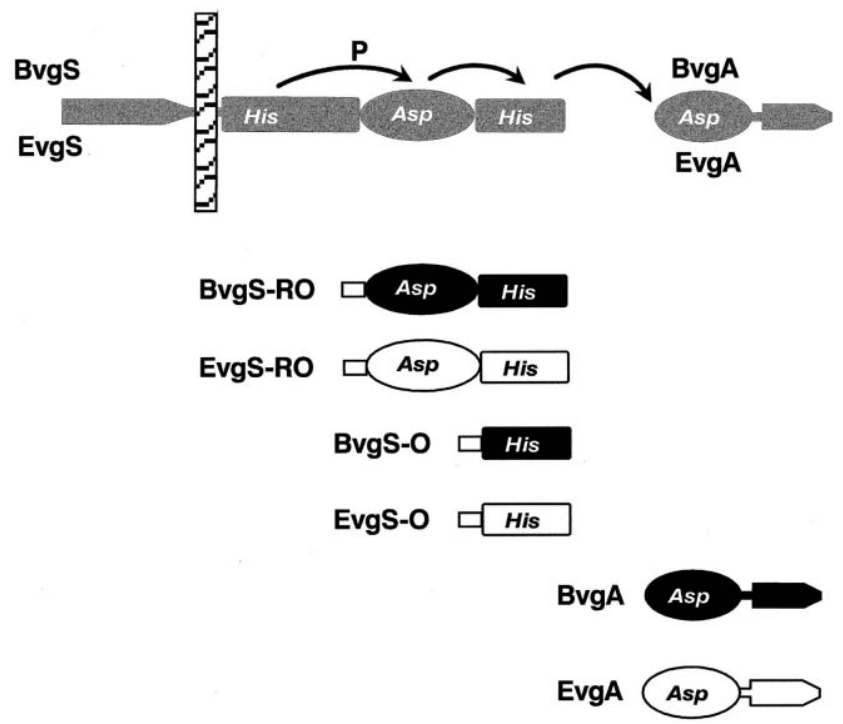

Fig. 1. Schematic presentation of the architecture of the proteins of the BvgAS and EvgAS two-component systems and their derivatives used in this study. At the top the domain structure and the phosphorelay within the wild type systems is depicted schematically. At the bottom the domain composition of the histidine kinase derived proteins BvgS-RO, BvgS-O, EvgS-RO and EvgS-O and of the effector proteins BvgA and EvgA is shown, respectively. The box in front of the histidine kinase derived proteins indicates the N-terminal His-tag.

viously described His-tagged signalling domains EvgS-RO, EvgS-O, BvgS-RO and BvgS-O [9,23] (Fig. 1) were isolated in mg quantities and subjected to biophysical and biochemical characterization methods including native gradient gel electrophoresis, gel permeation chromatography (GPC) and AUC. First, all protein samples were checked for purity and homogeneity by SDS-PAGE (data not shown) and mass spectrometric molecular mass determinations. The determined molecular masses of all proteins precisely matched the calculated masses from the DNA-translated amino acid sequences (Table 2). The mass spectrometric results, thus, proved the integrity of all proteins and constructs and confirmed the absence of high molecular mass contaminants in the protein samples.

Providing initial evidence for the dimeric status of EvgA, on native gradient gels the $22.69 \mathrm{kDa}$ protein migrated at a position of about $46 \mathrm{kDa}$ (Fig. 2, top). Under denaturing conditions the EvgA protein migrated at a position corresponding to its monomeric form, but a band at the position of the dimer could 

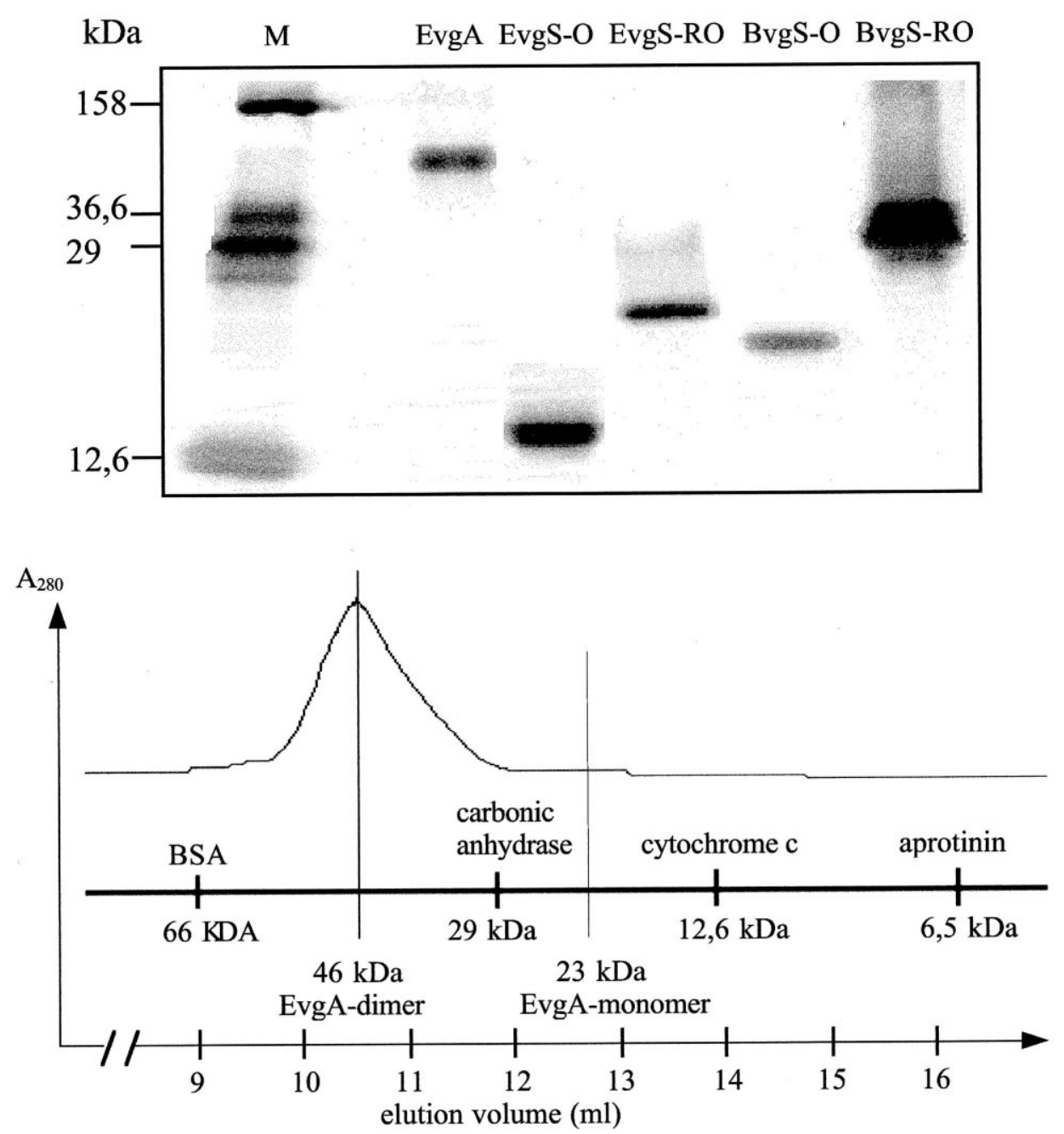

Fig. 2. Representative examples of native gradient gels and gel permeation experiments. (Top) A native gradient gel is shown with EvgA, EvgS-O, EvgS-RO, BvgS-O and BvgS-RO as samples. On the left the MW standard is shown. (Bottom) The elution profile of EvgA on a Superdex 75 column is shown. The elution positions of the MW standards and of EvgA are indicated. The dotted line marks the position where the EvgA monomer is expected.

still be detected (data not shown). In contrast to EvgA, on the native gradient gels the histidine kinase derived protein domains EvgS-RO, EvgS-O, BvgS$\mathrm{RO}$ and $\mathrm{BvgS}-\mathrm{O}$ migrated approximately at positions expected for the molecular weight of their monomeric forms or somewhat lower (Fig. 2, top).

GPC was carried out on a Superdex 75 column. EvgA eluted at an elution volume corresponding to $46 \mathrm{kDa}$, which is the molecular weight of the EvgA dimer. No EvgA protein could be detected at the position corresponding to its monomeric form (Fig. 2, bottom, and Table 3). Attempts to destabilize the dimer by the addition of $10 \mathrm{mM}$ EDTA to remove $\mathrm{Mg}^{2+}$ ions, $2 \mathrm{M}$ urea or lowering the $\mathrm{pH}$ to $3.8 \mathrm{did}$ not result in the dissociation of the EvgA dimer to its monomeric form, indicating a very strong association of the EvgA dimer (data not shown). The histidine kinase derived truncated proteins EvgS-RO, BvgS$\mathrm{RO}$, EvgS-O and BvgS-O eluted in a range corresponding to the molecular weight of their monomeric forms, although, for unknown reasons, BvgS-O repeatedly eluted at a position somewhat too high with regard to the molecular weight of its monomer (Table 3). Analysis of the dimerization state of BvgA using native gradient gels and the gel permeation experiments were hampered by the low solubility of this protein under the experimental conditions (data not shown) and were therefore not considered. 
Table 3

Determination of the molecular weight of signalling proteins by AUC a and GPC on a Superdex-75 column

\begin{tabular}{|c|c|c|c|c|c|}
\hline & $\bar{v}_{4^{\circ} \mathrm{C}}\left(\mathrm{ml} \mathrm{g}^{-1}\right)$ & $\begin{array}{l}\text { MW monomer } \\
(\mathrm{kDa})\end{array}$ & $\begin{array}{l}\text { MW measured by } \\
\text { AUC (kDa) }\end{array}$ & $\begin{array}{l}\text { MW measured by } \\
\text { GPC }(\mathrm{kDa})\end{array}$ & $\begin{array}{l}\text { Association } \\
\text { state }\end{array}$ \\
\hline BvgA & 0.736 & 22.95 & $45.1 \pm 3.1$ & n.d. ${ }^{\mathrm{b}}$ & dimer \\
\hline EvgA & 0.736 & 22.69 & $40.4 \pm 2.6$ & $46.0 \pm 0.5$ & dimer \\
\hline BvgS-RO & 0.727 & 31.89 & $22.9 \pm 5.7$ & $32.4 \pm 2.5$ & monomer \\
\hline EvgS-RO & 0.731 & 31.11 & $31.5 \pm 0.9$ & $31.9 \pm 2.0$ & monomer \\
\hline BvgS-O & 0.725 & 17.28 & $16.8 \pm 0.5$ & $24.0 \pm 1.2$ & monomer \\
\hline EvgS-O & 0.727 & 16.13 & $19.1 \pm 3.3$ & $19.5 \pm 0.8$ & monomer \\
\hline BvgS-RO+10 mM acetyl phosphate & 0.727 & 31.89 & $24.4 \pm 2.6$ & n.d. & monomer \\
\hline EvgS-RO+10 mM acetyl phosphate & 0.731 & 31.11 & $30.9 \pm 2.0$ & n.d. & monomer \\
\hline
\end{tabular}

${ }^{\mathrm{a}}$ The data were averages of 2-8 measurements at 10000 and $25000 \mathrm{rpm}$ and were determined as described in Section 2 . For typical data sets see Fig. 3. The parameter $\bar{v}_{4}{ }^{\circ}$ is the calculated partial specific volume at $4^{\circ} \mathrm{C}$ of a given protein.

bn.d., not done.

The molecular weight of the BvgA and EvgA proteins was measured by AUC to correspond to 45.1 $( \pm 3.1)$ and $40.4( \pm 2.6) \mathrm{kDa}$, respectively, with monomer protein concentrations of $10-20 \mu \mathrm{M}$. Since the calculated molecular weights of the monomers are 22.9 and $22.4 \mathrm{kDa}$, respectively, these data demonstrate that both proteins, BvgA and EvgA, formed a dimer in solution. As an example, in Fig. 3A a data set for the EvgA protein is shown, which demonstrates a very good fit to a single exponential equation. As exclusively dimeric proteins could be detected by AUC at the protein concentrations indicated, the dimer dissociation constant can be deduced to be significantly below $1 \mu \mathrm{M}$, a limit recently also estimated for the NtrC dimer, a two-component effector protein involved in nitrogen regulation [32]. The analysis of the sensor derived domains resulted in each case in molecular weight determinations corresponding to their monomeric forms (Table 3).

In conclusion, with different methods consistent results were obtained which demonstrate that the non-phosphorylated effector proteins EvgA and BvgA are dimers in solution, whereas EvgS and BvgS derived receiver and HPt domains are monomers.

\subsection{Acetyl phosphate mediated phosphorylation of receiver domains and analysis of its impact on the oligomerization properties of sensor derived signalling modules}

In several cases, it was shown that phosphorylation of a receiver domain can induce cooperative binding to DNA or dimerization of the respective protein [12-16]. Accordingly, we attempted to analyze a possible impact of acetyl phosphate on the oligomerization state of the monomeric sensor derived receiver containing proteins. First we compared the phosphorylation properties of the EvgS-RO and BvgS-RO proteins and of the effector proteins EvgA and BvgA. All four proteins were labeled efficiently in the presence of acetyl $\left[{ }^{32} \mathrm{P}\right]$ phosphate (Fig. 4) but not of $\left[{ }^{33} \mathrm{P}\right] \gamma \mathrm{ATP}$ (data not shown). In agreement with previous results, no phosphorylation of the isolated HPt domains, BvgS-O and EvgS-O could be observed (data not shown), but addition of these HPt domains to the BvgS-RO and EvgS-RO proteins in the presence of acetyl $\left[{ }^{32} \mathrm{P}\right]$ phosphate resulted in phosphotransfer to the HPt domains (Fig. 4). In general, the phosphorylation patterns of the BvgA, EvgA, BvgS-RO and EvgS-RO proteins were very similar reaching a maximal phosphorylation level of the proteins after 20-30 min (Fig. 4). However, phosphorylation of the BvgS-RO and EvgS-RO proteins harboring the intermediate receiver domains appeared to be somewhat faster than phosphorylation of the effector proteins (Fig. 4).

After the demonstration of their autophosphorylation ability we attempted to detect the impact of phosphorylation on the oligomerization status of the truncated sensor derived proteins. Since acetyl phosphate does not interfere with the absorbance at $280 \mathrm{~nm}$, the ultracentrifugation experiments with the BvgS-RO and EvgS-RO proteins could be performed in the presence of $10 \mathrm{mM}$ acetyl phosphate and $10 \mathrm{mM} \mathrm{MgCl}_{2}$ to allow chemical phosphorylation. 

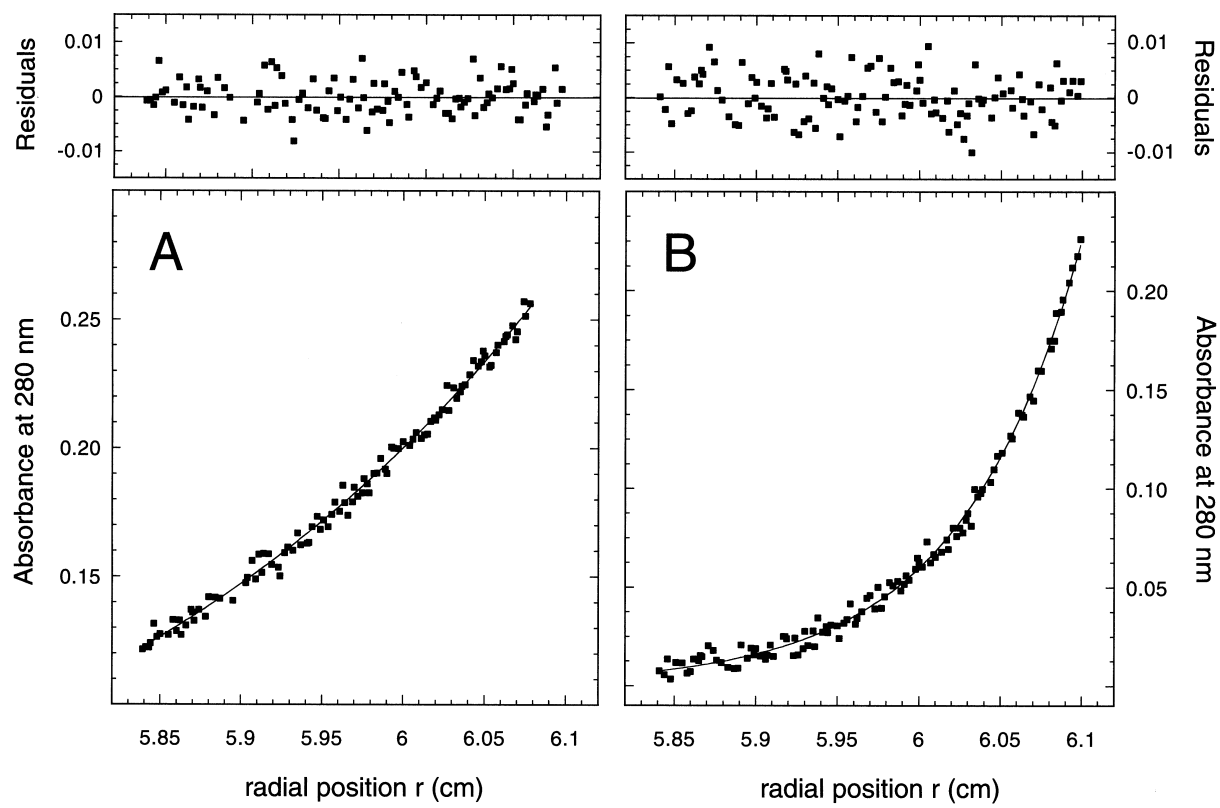

Fig. 3. Examples of AUC equilibrium runs with EvgA and EvgS-RO. Equilibrium was reached after 18-22 h. In the bottom part of the figure the measured absorbance at $280 \mathrm{~nm}$ versus the radial position (distance to the center of the rotor) is shown. The top part of the figure gives the residuals to the fit expressed as the difference between experimental and fitted values. Only every third data point is shown. (A) EvgA protein at a concentration of $17 \mu \mathrm{M}$ monomer (calculated MW $22.4 \mathrm{kDa}$ ) centrifuged at $10000 \mathrm{rpm}$. For the data presented in this figure a MW of $41.3 \mathrm{kDa}$ was determined from the fit. For average values see Table 2. (B) EvgS-RO analyzed in the presence of $10 \mathrm{mM}$ acetyl phosphate and $10 \mathrm{mM} \mathrm{MgCl} 2$ to allow chemical phosphorylation of the protein. Centrifugation of a $10 \mu \mathrm{M}$ protein solution was carried out at $25000 \mathrm{rpm}$. In this experiment a MW of $28.8 \mathrm{kDa}$ was determined for the protein as compared to the value of $31.8 \mathrm{kDa}$ calculated for a monomer. Average values are given in Table 2.

However, both proteins remained in their monomeric form and no indication of a phosphorylation induced dimerization/oligomerization was obtained for BvgS$\mathrm{RO}$ and EvgS-RO as well as for BvgS-O and EvgS-O (Table 3 and Fig. 3B).

\subsection{Biotinylation of the EvgA protein and the EvgS HPt domain}

SPR has recently been applied successfully to monitor interactions of two-component proteins [34,35]. In the present study we started to use this technology to analyze the interaction between the EvgA effector protein and its histidine kinase EvgS and to gain further insight in the oligomerization status of these proteins. For this purpose, the EvgS derived HPt domain (EvgS-O) and the receiver containing EvgSRO protein were chemically modified by biotinylation. In order to identify the preferential modification sites, aliquots of the biotinylated proteins were subjected to mass spectrometric analyses after tryptic digestion. Each incorporated biotinyl group adds a mass increment of $339.5 \mathrm{Da}$ which is easily detectable by mass spectrometry. In general, selectivity of the modification reaction depends on the protein structure and the reaction conditions, particularly to the molar ratios of the reactants $[36,37]$. Hence, instead of seeking conditions that allowed complete modification of Lys-residues, we established conditions by which only particular Lys residues were modified due to their distinctive accessibility. With this goal we deliberately took into account that partial modification was achieved. Mass spectrometric peptide mapping of biotinylated (protein/reagent ratio was 1:5) and tryptically digested EvgS-O showed a mixture of peptide ion signals (Fig. 5A). In this mixture an ion signal was observed at $\mathrm{m} / \mathrm{z} 3679$ for unmodified peptide (29-59). This peptide possessed two lysine residues, Lys-36 and Lys-59. The latter residue served as a cleavage site for trypsin. In addition, peptide (2959) was also found biotinylated, as was evident from an ion signal observed at $\mathrm{m} / \mathrm{z}$ 4019. Again, Lys-59 in this peptide was recognized as a cleavage site by trypsin. Tryptic cleavage is possible only at unmodi- 

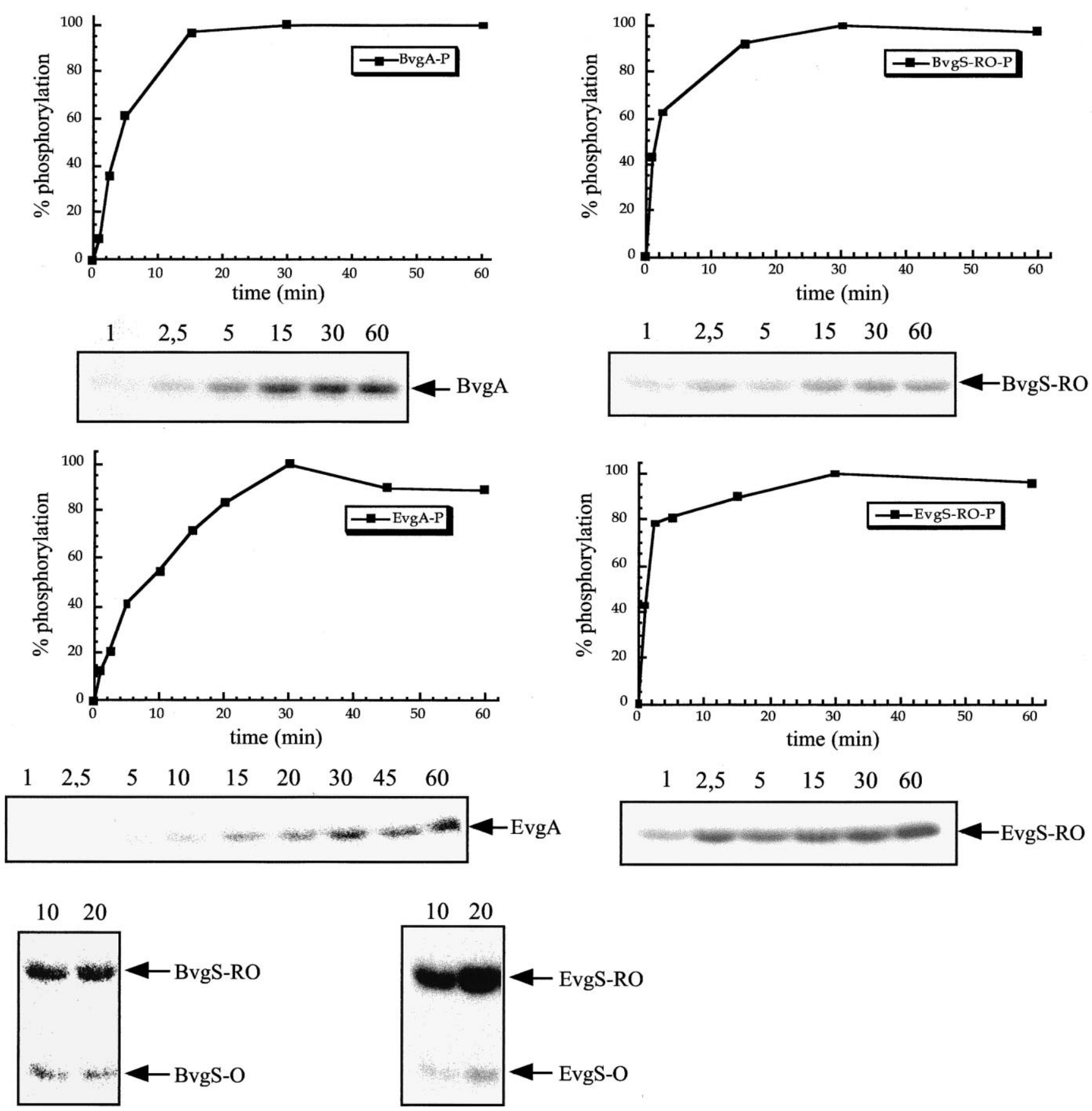

Fig. 4. The kinetics of autophosphorylation of the effector proteins BvgA and EvgA and of the histidine kinase derived proteins BvgS-RO and EvgS-RO in the presence of acetyl $\left[{ }^{32} \mathrm{P}\right]$ phosphate are shown. In each panel on the bottom the original gel is shown, whereas in the graph on the top the results of the quantitation of the signal are shown. In the lower part of the figure, transphosphorylation in the presence of acetyl phosphate of the BvgS-O and EvgS-O proteins mediated by the BvgS-RO and EvgS-RO proteins, respectively, is shown. 


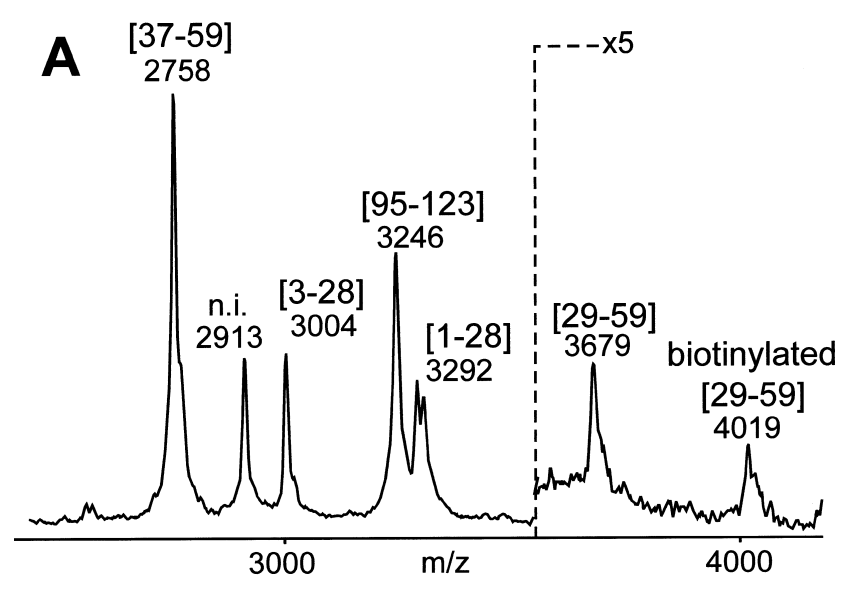

B

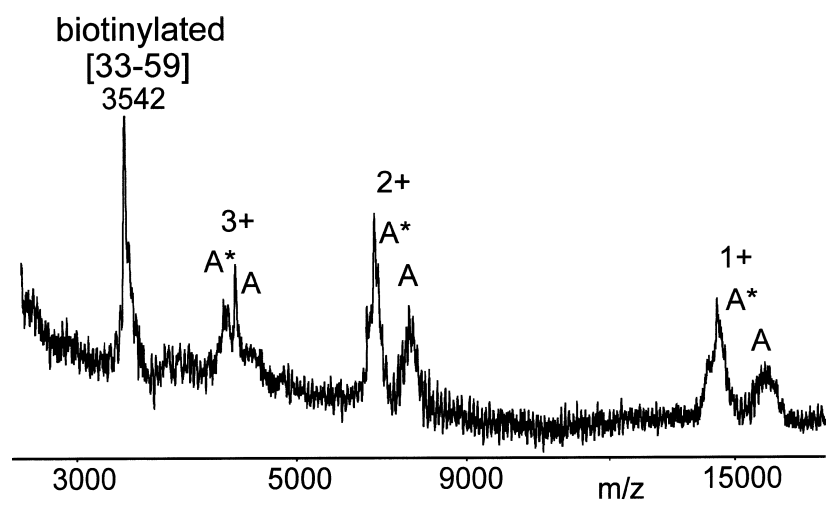

Fig. 5. Mass spectrometric identification of the biotinylation site in EvgS-O. (A) The MALDI-spectrum (range: $\mathrm{m} / \mathrm{z} 2500$ 4500) obtained from biotinylated and tryptically digested EvgS$\mathrm{O}$ shows a mixture of peptide ion signals. The peptide ion signal that contains the biotinylated residue K-36 is indicated. (B) MALDI-MS analysis of biotinylated EvgS-O peptide obtained by digestion with endoproteinase Asp-N and subsequent affinity isolation using avidin Sepharose. After extensive washing a single biotinylated EvgS-O peptide remained and was detected together with ion signals belonging to avidin. A indicates avidin; $A^{*}$ represents a deglycosylated form of avidin. Numbers give $\mathrm{m} / \mathrm{z}$ values. Numbers in parentheses refer to amino acid positions as in the EvgS-O sequence. HCCA was used as a matrix.

fied lysine residues. Hence, mass spectrometry and the enzymatic cleavage specificity located the predominant biotinylation site at Lys-36. Lysine residue Lys-36 in the HPt domain (EvgS-O) corresponds to Lys-1092 in the EvgS wild type sequence.

Biotinylation at Lys-36 was independently proven by using mass spectrometry together with specific biotin affinity isolation of proteolytic peptides after digestion of biotinylated EvgS-O (protein/reagent ra- tio was 1:2). After digestion with Asp-N protease the peptide mixture was incubated with Sepharose coupled avidin. As avidin exhibits a very strong binding affinity to biotin, only biotinylated peptides remained in the Sepharose precipitate whereas all other peptides were removed in the supernatant by extensive washing. After transferring the Sepharose avidin/biotinylated peptide complex to the MALDIMS target and after addition of the matrix, spectra were recorded in which the only observed components were ions from singly and multiply charged avidin and from biotinylated EvgS-O peptide (3359) (Fig. 5B). Similar experiments were carried out with the biotinylated EvgS-RO protein and showed that this signalling module was modified preferentially within its receiver domain at the conserved lysine residue which corresponds to K1059 in wild type EvgS (data not shown).

\subsection{Interaction between $\operatorname{Evg} A$ and the EvgS HPt domain}

To characterize the interaction of the soluble EvgA protein with the immobilized histidine kinase derived protein domains using the BIAcore biosensor system, increasing EvgA concentrations were added to the sensor chip ranging from 0.11 to $11 \mu \mathrm{M}$. Fig. 6 shows the SPR signals expressed in arbitrary RU obtained at different EvgA concentrations with the immobilized EvgS-O protein. The affinity constant of the EvgS-O/EvgA interaction was estimated to be $1.24 \times 10^{6}\left( \pm 1.3 \times 10^{4}\right) \mathrm{M}^{-1}$ demonstrating a relatively weak but specific interaction of these proteins. Evidence for the stoichiometry of the interaction partners could be deduced from the SPR signals, as the relative amount of the two protein partners can be determined considering the ratio of the SPR signal and the molecular weight of the respective proteins. The respective values were found to be 0.065 and 0.038 for EvgS-O and EvgA, respectively, indicating a 1:2 stoichiometry. The same affinity constant and stoichiometry of the interacting partners were obtained with the immobilized EvgS-RO protein (data not shown). This stoichiometry is in agreement with the results reported above indicating that EvgA forms dimers in solution. The effect of phosphorylation of the EvgS-RO protein on its interaction with EvgA could not be further analyzed, because, as 


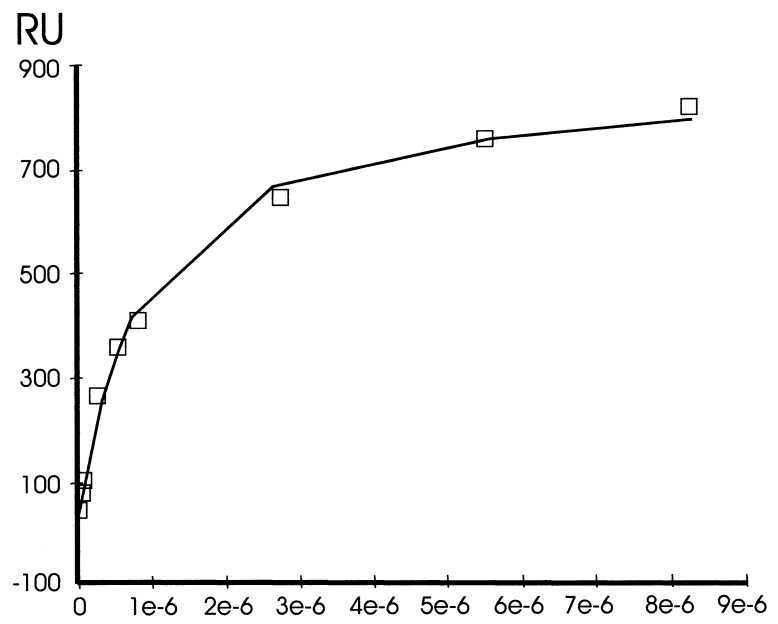

[EvgA]

Fig. 6. Graphic representation of the RU detected with EvgS-O immobilized on the BIAcore sensor chip after addition of increasing concentrations of EvgA.

pointed out above, biotinylation of the EvgS-RO protein occurred at the crucial lysine residue within the receiver domain. The interaction of the BvgS-O and $\mathrm{BvgA}$ proteins could not be analyzed in this system due to solubility problems with the BvgA protein under the given experimental conditions (data not shown).

\section{Discussion}

Phosphorylation of the effector proteins by their cognate histidine kinases is the crucial event which regulates their biological activity [4]. Although the structural consequences of phosphorylation on the effector proteins are not well understood, in several cases it has been observed that phosphorylation induces dimerization and/or cooperative binding to DNA. [12-16]. Thus, effector proteins engaged in the activation of transcription are at least in part activated by increasing their affinity to their respective target sites. Dimerization/oligomerization of signalling domains may therefore be an important feature involved in the phosphorylation dependent regulation of the biological activity of the effector proteins. In the present study we analyzed the oligomerization state of the non-phosphorylated BvgA and EvgA effector proteins. As the multi-domain
BvgS and EvgS histidine kinases are integral membrane proteins and as such difficult to study, we analyzed several truncated derivatives lacking the transmembrane domains of the two proteins. Previously we have shown that the purified response regulators and also the truncated proteins derived from the histidine kinases retain important activities such as phosphorylation properties, DNA binding activity or the capability of transcomplementation in vivo $[9,18,23]$. As indicated by mass spectrometry the purified EvgA and BvgA proteins were not phosphorylated at the time of their analysis (Table 2). The lack of phosphorylation corresponds to the fact that, in the case of $\mathrm{BvgA}$, the cognate histidine kinase $\mathrm{BvgS}$ is not present in the overexpressing $E$. coli strain, whereas in the case of EvgA its overexpression was achieved under growth conditions which inactivated the EvgS histidine kinase (addition of $\mathrm{MgSO}_{4}$ to the medium) [8].

\subsection{Oligomerization state of signalling proteins derived from the BvgAS and EvgAS systems}

Consistently, the AUC experiments, the native gradient gels and gel permeation experiments demonstrated that the non-phosphorylated effector proteins EvgA and BvgA are dimers in solution. These results are in agreement with preliminary data published previously which also indicated the occurrence of dimeric forms of BvgA in vivo [9,19,20]. Due to extensive sequence similarities the EvgA and BvgA proteins are classified in the so-called NarL subfamily of effector proteins. Although previously considered to be a monomer in solution which dimerizes upon phosphorylation, the recent comparison of several different crystal structures of the closely related NarL effector protein indicated that also NarL may have the tendency to form homodimers in the nonphosphorylated form $[38,39]$. In the future, it will be interesting to analyze the oligomerization state of these proteins after phosphorylation and/or after binding of DNA. In contrast to the effector proteins, no indication for a dimeric state of the nonphosphorylated histidine kinase derived domains BvgS-RO, BvgS-O, EvgS-RO and EvgS-O could be found.

To analyze whether phosphorylation may induce oligomerization of the histidine kinase derived pro- 
teins, we performed ultracentrifugation experiments in the presence of acetyl phosphate. This was possible, because, as discussed below, the histidine kinase derived intermediate receiver domains are able to autophosphorylate in vitro in the presence of acetyl phosphate (Fig. 4). Under the experimental conditions of ultracentrifugation acetyl phosphate is expected to be stable. Hence, differences in the sedimentation properties of the proteins in its absence or presence should be detectable. However, no indication for a phosphorylation dependent dimerization of these domains could be observed (Fig. 3B). Therefore, it is likely that these protein domains do not oligomerize upon phosphorylation.

\subsection{Phosphorylation of receiver domain containing proteins}

In the case of the receiver domains of effector proteins it was shown that they harbor the enzymatic activity for phosphorylation and, accordingly, are able to phosphorylate in the presence of compounds such as acetyl phosphate. Here we show, that the intermediate receiver domains derived from the unorthodox histidine kinases EvgS and BvgS are also able to phosphorylate using ${ }^{32} \mathrm{P}$-labelled acetyl phosphate as a substrate. As shown in Fig. 4, the phosphorylation properties of the effector proteins and of the proteins containing the intermediate receiver domains are very similar. Note, however, that the interpretation of the phosphorylation patterns must take into account that the visible phosphorylation signals of the EvgS-RO and BvgS-RO proteins derive exclusively from the phosphohistidine of their HPt domains. It had already been reported that the phosphoaspartates of the EvgS and BvgS receiver domains are very unstable and can not be visualized directly after SDS-PAGE $[23,40]$. The HPt domains require phosphorylation of the intermediate receiver domains prior to their phosphorylation. Therefore, the phosphorylation signals detected with the EvgSRO and BvgS-RO proteins reflect the phosphorylation of their receiver domains only indirectly (Fig. 4) $[9,40]$. One other intermediate receiver domain of a multi-domain histidine kinase, DokA of Dictyostelium discoideum, has recently been shown to be able to autophosphorylate [41]. It is likely that, similar to the receiver domains of effector proteins, the inter- mediate receiver domains present in unorthodox histidine kinases generally harbor the enzymatic activity for the phosphotransfer reaction.

\subsection{Characterization of the EvgA-EvgS interaction by $S P R$}

The biosensor experiments allowed preliminary characterization of the interaction of EvgA with the EvgS HPt domain (Fig. 6). The affinity constant was calculated to be about $1.24 \times 10^{6} \mathrm{M}^{-1}$, which indicates a relatively weak interaction of the two proteins as compared to results obtained with the CheA/ CheY and VanS/VanR two-component systems $[34,42]$. It is possible that this relatively low affinity may be the consequence of a lack of phosphorylation of the histidine kinase derived protein domains in our biosensor experiments. In fact, an impact of phosphorylation on the stability of the complex between the histidine kinase and the effector protein was recently demonstrated for the CheA/CheY proteins [34] and, accordingly, will be analyzed in the future with the Evg and Bvg proteins. Alternatively, it is possible that an efficient interaction between EvgA and EvgS requires an EvgS dimer. All histidine kinases analyzed so far including BvgS are able to form homodimers. Homodimerization is mediated by the transmitter domains of the histidine kinases and appears to be independent of the phosphorylation state of the proteins $[9,28,43,44]$. As shown here, the EvgS-RO and EvgS-O proteins lacking the EvgS transmitter domain are monomers, a conformation which may not allow a high affinity interaction of the proteins.

Recently, Utsumi et al. attempted to characterize the oligomerization state of EvgA using SPR. For this purpose they coupled EvgA to the sensor chip and added soluble non-phosphorylated or phosphorylated EvgA. However, as no resonance signal could be detected, it was concluded that EvgA is a monomer independent of its phosphorylation state [35]. In marked contrast, AUC, native gradient gels and the gel permeation experiments described here consistently demonstrate that non-phosphorylated EvgA is a dimer in solution. Moreover, the biosensor experiments revealed that EvgA interacts as a dimer with the EvgS HPt module coupled to the sensor chip. There are several possible explanations for the 
different results. We show that EvgA is a dimer in solution and as such may not interact anymore with EvgA protein coupled to the sensor chip. Also, as shown here for the EvgS-RO protein, chemical modification of the protein required for its immobilization on the sensor chip can significantly interfere with important functional parts of a protein. In the case of the EvgS-RO protein a strictly conserved lysine residue important for the structural integrity of the receiver domain and for phosphorylation induced conformational changes is the main target of biotinylation (Fig. 5). As the structure of the chemically modified EvgA proteins was not characterized in the previous work, it is possible that the corresponding lysine residue within its receiver domain was also a preferred target for biotinylation of EvgA and may therefore interfere strongly with the structure of the immobilized protein and with its biophysical properties.

\section{Acknowledgements}

We thank Dagmar Beier for critical reading of the manuscript. This work was supported by grant Gr1243/2-4 from the Deutsche Forschungsgemeinschaft (Priority program 'Regulatory Networks in Bacteria') and by the Fonds der Chemischen Industrie.

\section{References}

[1] W.F. Loomis, A. Kuspa, G. Shaulsky, Curr. Opin. Microbiol. 1 (1997) 643-648.

[2] J.L. Appleby, J.S. Parkinson, R.B. Bourret, Cell 86 (1996) 845-848.

[3] A.-L. Perraud, V. Weiss, R. Gross, Trends Microbiol. 7 (1999) 115-120.

[4] J.B. Stock, A.M. Stock, J.M. Mottonen, Nature 344 (1990) 395-400.

[5] W.R. McCleary, J.B. Stock, A.J. Ninfa, J. Bacteriol. 175 (1993) 2793-2798.

[6] J. Feng, M.R. Atkinson, W.R. McCleary, J.B. Stock, B.L. Wanner, A.J. Ninfa, J. Bacteriol. 174 (1992) 6061-6070.

[7] K. Ishige, S. Nagasawa, S. Tokishita, T. Mizuno, EMBO J. 13 (1994) 5195-5202.

[8] R. Utsumi, S. Katayama, M. Taniguchi, T. Horie, M. Ikeda, S. Igaki, H. Nakagawa, A. Miwa, H. Tanabe, M. Noda, Gene 140 (1994) 73-77.
[9] D. Beier, B. Schwarz, T.M. Fuchs, R. Gross, J. Mol. Biol. 248 (1995) 596-610.

[10] M.A. Uhl, J.F. Miller, EMBO J. 15 (1996) 1028-1036.

[11] B. Arico, J.F. Miller, C. Roy, S. Stibitz, D. Monack, S. Falkow, R. Gross, R. Rappuoli, Proc. Natl. Acad. Sci. USA 86 (1989) 6671-6675.

[12] U. Fiedler, V. Weiss, EMBO J. 14 (1995) 3696-3705.

[13] K.J. Huang, C.Y. Lan, M.M. Igo, Proc. Natl. Acad. Sci. USA 94 (1997) 2828-2832.

[14] V. Weiss, F. Claverie-Martin, B. Magasanik, Proc. Natl. Acad. Sci. USA 89 (1992) 5088-5092.

[15] J. Li, S. Kustu, S. Stewart, J. Mol. Biol. 241 (1994) 150-165.

[16] W.R. McCleary, Mol. Microbiol. 20 (1996) 1155-1163.

[17] J.B. Stock, Science 274 (1996) 370-371.

[18] D. Beier, H. Deppisch, R. Gross, Mol. Gen. Genet. 252 (1996) 169-176.

[19] V. Scarlato, A. Prugnola, B. Arico, R. Rappuoli, Proc. Natl. Acad. Sci. USA 87 (1990) 6753-6757.

[20] P.E. Boucher, F.D. Menozzi, C. Locht, J. Mol. Biol. 241 (1994) 363-377.

[21] F. Baneyx, G. Georgiou, J. Bacteriol. 173 (1991) 26962703.

[22] T.N. Belev, M. Singh, J.E.G. McCarthy, Plasmid 26 (1991) 147-150.

[23] A.-L. Perraud, B. Kimmel, V. Weiss, R. Gross, Mol. Microbiol. 27 (1998) 875-887.

[24] D.C. Schriemer, L. Li, Anal. Chem. 68 (1996) 3382-3387.

[25] D.C. Schriemer, T. Yalcin, L. Li, Anal. Chem. 70 (1998) 1569-1575.

[26] T. Fligge, K. Bruns, M. Przybylski, J. Chromatogr. B Biomed. Sci. Appl. 706 (1998) 91-100.

[27] M. Bantscheff, V. Weiss, M.O. Glocker, Biochemistry 38 (1999) 11012-11020.

[28] R. Heermann, K. Altendorf, K. Jung, Biochim. Biophys. Acta 1415 (1998) 114-124.

[29] E.R. Stadtman, Methods Enzymol. 3 (1957) 228-231.

[30] S. Bouche, E. Klauck, D. Fischer, M. Lucassen, K. Jung, R. Hengge-Aronis, Mol. Microbiol. 27 (1998) 787-795.

[31] U.K. Laemmli, Nature 227 (1970) 680-685.

[32] K. Rippe, N. Mücke, A. Schulz, J. Mol. Biol. 278 (1998) 915-933.

[33] B.J. Shen, T. Hage, W. Sebald, Eur. J. Biochem. 240 (1996) 252-261.

[34] S.C. Schuster, R.V. Swanson, L.A. Alex, R.B. Bourret, M.I. Simon, Nature 365 (1993) 343-347.

[35] H. Tanabe, T. Masuda, Y. Yamasaki, A. Katoh, S. Yoshioka, R. Utsumi, Biosci. Biotechnol. Biochem. 62 (1998) 7882.

[36] M.O. Glocker, C. Borchers, W. Fiedler, D. Suckau, M. Przybylski, Bioconjug. Chem. 5 (1994) 583-590.

[37] M. Kalkum, M. Przybylski, M.O. Glocker, Bioconjug. Chem. 9 (1998) 226-235.

[38] I. Baikalov, I. Schröder, M. Kaczor-Grzeskowiak, K. Grzeskowiak, R.P. Gunsalus, R.E. Dickerson, Biochemistry 35 (1996) 11053-11061.

[39] I. Baikalov, I. Schröder, M. Kaczor-Grzeskowiak, D. Cas- 
cio, R.P. Gunsalus, R.E. Dickerson, Biochemistry 37 (1998) 3665-3676.

[40] M.A. Uhl, J.F. Miller, J. Biol. Chem. 271 (1996) 3317633180 .

[41] S.S. Schuster, A.A. Noegel, F. Oehme, G. Gerisch, M.I. Simon, EMBO J. 15 (1996) 3880-3889.
[42] S.L. Fisher, S.K. Kim, B.L. Wanner, C.T. Walsh, Biochemistry 35 (1996) 4732-4740.

[43] Y. Hidaka, H. Park, M. Inouye, FEBS Lett. 400 (1997) 238242.

[44] S.Q. Pan, T. Charles, S. Jin, Z.L. Wu, E.W. Nester, Proc. Natl. Acad. Sci. USA 90 (1993) 9939-9943. 